\title{
Gene-centromere mapping in meiotic gynogenetic European seabass
}

\author{
Münevver Oral ${ }^{1 \dagger}$, Julie Colléter ${ }^{2,3 \dagger}$, Michaël Bekaert ${ }^{1 \dagger}$, John B Taggart ${ }^{1}$, Christos Palaiokostas ${ }^{1}$, \\ Brendan J. McAndrew ${ }^{1}$, Marc Vandeputte ${ }^{3,4}$, Béatrice Chatain ${ }^{3}$, Heiner Kuhl ${ }^{5}$, Richard Reinhardt ${ }^{6}$, \\ Stefano Peruzzi ${ }^{7}$ and David J Penman ${ }^{1 *}$ (D)
}

\begin{abstract}
Background: Fully isogenic lines in fish can be developed using "mitotic" gynogenesis (suppression of first zygotic mitosis following inactivation of the sperm genome). However, genome-wide verification of the steps in this process has seldom been applied. We used ddRADseq to generate SNP markers in a meiotic gynogenetic family of European seabass (Dicentrarchus labrax): (i) to verify the lack of paternal contribution in a meiotic gynogenetic family; (ii) to generate a gene-centromere map from this family; (iii) to identify telomeric markers that could distinguish mitotic gynogenetics from meiotic gynogenetics, which sometimes arise spontaneously in mitotic gynogenetic families.

Results: From a single meiotic gynogenetic family consisting of 79 progeny, 42 million sequencing reads (Illumina, trimmed to 148 bases) resolved 6866 unique RAD-tags. The 340 male-informative SNP markers that were identified confirmed the lack of paternal contribution. A gene-centromere map was constructed based on 804 female-informative SNPs in 24 linkage groups $(2 n=48)$ with a total length of $1251.02 \mathrm{cM}$ (initial LG assignment was based on the seabass genome assembly, dicLab v1). Chromosome arm structure could be clearly discerned from the pattern of heterozygosity in each linkage group in 18 out of 24 LGs: the other six showed anomalies that appeared to be related to issues in the genome assembly.

Conclusion: Genome-wide screening enabled substantive verification of the production of the gynogenetic family used in this study. The large number of telomeric and subtelomeric markers with high heterozygosity values in the meiotic gynogenetic family indicate that such markers could be used to clearly distinguish between meiotic and mitotic gynogenetics.
\end{abstract}

Keywords: Dicentrarchus labrax, Meiotic gynogenesis, Isogenic lines, ddRAD seq, Genetic map, Gene-Centromere map, Aquaculture

\section{Background}

Polyploidy has occurred during evolution of various fish groups [1] while gynogenesis is a natural form of reproduction in some species [2]. Spontaneous polyploids have also been observed in both wild and farmed fish [1]. Induced chromosome set manipulation is a methodology that has been exploited over a long period in fish research [3-7]. The ability to retain the second polar body post-fertilisation and/or suppress first cell

* Correspondence: d.j.penman@stir.ac.uk

${ }^{\dagger}$ Equal contributors

${ }^{1}$ Institute of Aquaculture, School of Natural Sciences, University of Stirling,

FK9 4LA Stirling, Scotland, UK

Full list of author information is available at the end of the article division by temperature, chemical or pressure shocks, coupled with the relative ease of gamete inactivation by irradiation has led to its widespread use. The various chromosome sets that can be generated (haploids, triploids, tetraploids, androgenetics, meiotic or mitotic gynogenetics) have been exploited in a wide range of studies including gene mapping $[8,9]$, genome assembly $[10,11]$, construction of isogenic lines $[12,13]$ and production of sterile farm fish $[14,15]$.

European seabass is an important mariculture species, extensively farmed in the Mediterranean basin. The need to develop genetic and genomic resources to underpin future development of this species is clearly recognised, 
and has resulted in the production of a first draft genome assembly [16], a number of linkage maps [17-19] and a radiation hybrid panel [20]. A further key resource would be the development of isogenic lines through androgenesis [21] or mitotic gynogenesis [22]. These have not been successfully established yet, despite significant efforts [21-24].

Though widely practised, there are a number of technical pitfalls that can impact the effectiveness of induced gynogenesis and androgenesis. For example, there can be a potential genetic contribution from the irradiated gamete source, this being associated with poorly optimised protocols leading to incomplete inactivation [2]. Furthermore, the efficiency of protocols designed to retain chromosome sets post fertilisation/activation can also be severely affected by gamete quality and slight alterations in the timing and intensity of the applied shock $[22,25,26]$. Spontaneous retention of the second polar body [27, 28] may also generate additional unexpected (and unwanted) ploidy states. One of the bottlenecks in production of isogenic lines through mitotic gynogenesis is spontaneous meiotic gynogenetics [29], which have some level of heterozygosity through retention of the second polar body, and need to be detected and eliminated from putative mitotic gynogenetic fish for the reliable production of isogenic lines in the subsequent generation.

Throughout the development of the technology, genetic markers have been used to monitor the effectiveness of the procedure. To date this has generally involved screening with a small panel of available markers, to confirm the presence/absence of particular parental chromosomal sets. These markers include pigmentation genes, allozymes, multilocus minisatellites and microsatellites [2]. While this approach can give an indication as to the effectiveness of the treatment, it is relatively insensitive for detection and quantification of potential instances of aneuploidy. Another limitation to using a small number of markers is that those that happen to be located close to centromeric regions will be compromised with respect to their ability to detect crossover events. This is a key requirement, for example, for differentiating between mitotic and meiotic gynogenetics; i.e. informative (heterozygous in the dam) telomeric markers will be heterozygous in meiotic gynogenetics and homozygous in mitotic gynogenetics, while informative centromeric markers will largely be homozygous in both types.

The advent of genotyping by sequencing approaches that exploit next generation sequencing technologies [30] permits the simultaneous discovery and screening of large numbers of single nucleotide polymorphisms (SNPs) per individual at a realistic cost. This provides an opportunity to more accurately assess the effectiveness of various elements of chromosomal set manipulation procedures. In this study SNPs generated by double digest restriction associated DNA (ddRAD) sequencing (ddRAD seq; [31]) were employed to comprehensively examine parental genetic contributions in an experimentally generated meiotic gynogenetic family of European seabass, Dicentrarchus labrax. The main objectives of the study were to (i) look for potential paternal contribution from UV-irradiated sperm; (ii) generate a SNP locus centromere map; and iii) screen informative (female heterozygous) markers for their potential to distinguish between mitotic and meiotic gynogenetics.

\section{Methods}

\section{Production of mapping family - Meiotic gynogenetics}

The meiotic gynogenetic seabass family was produced at the Ifremer Experimental Aquaculture Station (Palavasles-Flots, France), using parent fish from a West Mediterranean broodstock population. Broodstock were aged 4 to 6 years and weighed 1 to $5 \mathrm{~kg}$, and were kept in recirculating systems $\left(8 \mathrm{~m}^{3}\right.$ tanks, rate of $\mathrm{O}_{2}$ enriched water renewal $250 \mathrm{Lh}^{-1}$, constant low aeration) maintained under natural conditions of temperature and photoperiod $\left(43^{\circ} 31^{\prime} 40 \mathrm{~N}, 3^{\circ} 55^{\prime} 37 \mathrm{E}\right)$ and fed commercial diets (NeoRepro, Le Gouessant, France). Spermiating males were identified by gentle abdominal pressure and held in a handling tank. Female maturation stage was assessed from ovarian biopsies obtained by introducing a thin catheter (Pipelle de Cornier, Laboratoire CCD, Paris, France) into the genital orifice. Females at the correct stage of development received a single dose $\left(10 \mu \mathrm{g} \cdot \mathrm{kg}^{-1}\right)$ of Luteinizing Hormone Releasing Hormone analogue (LHRHa, Sigma, France) in order to induce final maturation and ovulation. The UV irradiation device, used to inactivate the paternal genome, comprised of eight UV lamps (12 W, $254 \mathrm{~nm}$, Vilber-Lourmat, Marne-la-Vallée, France) fixed above and below (four lamps each) a quartz plate which was mechanically agitated to stir sperm samples throughout irradiation. Diluted sperm $(5 \mathrm{~mL})$ from a single male (diluted 1:20, $v / \mathrm{v}$ in artificial extender SGSS [Seabass Gamete Short term Storage] made of StorFish [IMV Technologies, France] complemented with pyruvate and glutamine at 0.6 and $3 \mathrm{mg} / \mathrm{ml}^{-1}$ respectively [C. Fauvel, pers. comm.]) was irradiated in an $8.5 \mathrm{~cm}$ diameter quartz petri dish for $8 \mathrm{~min}$ to apply a total dose of $326 \mathrm{~mJ} / \mathrm{cm}^{2}$ [23].

The irradiated sperm were added to $125 \mathrm{~mL}$ of eggs (untreated, good quality) collected from a single female and then $125 \mathrm{~mL}$ of seawater was added to initiate fertilisation. A pressure shock of 8500 psi and 2 min duration was applied, starting at $6 \mathrm{~min}$ after fertilisation, to restore diploidy via retention of the second polar body [23]. All procedures were performed under total darkness in a temperature-controlled room maintained at $14{ }^{\circ} \mathrm{C}$. Eggs were incubated in $40 \mathrm{~L}$ tanks in a dedicated recirculating water system (temperature $14-14.5{ }^{\circ} \mathrm{C}$; salinity $35-36 \%$ ) until hatching. All tanks were maintained in darkness until 
sampling. Ten days after hatching, a subset of 80 larvae were fixed in $99 \%$ ethanol; fin tissue from parents was also fixed in ethanol.

\section{DNA preparation}

DNA was extracted from all 80 offspring (entire larva) and both parents (fin tissue) using a commercial salting out kit (REALpure DNA extraction kit; REAL Laboratories, Durviz, Spain) according to the manufacturer's protocol. This included the recommended RNase incubation step to reduce RNA contamination in the final product. The DNA concentration and purity of each sample was assessed by spectrophotometry (Nanodrop, Thermo Scientific, UK), while its integrity was assessed by $0.7 \%$ agarose gel electrophoresis. Each sample was then preliminarily diluted to $\mathrm{c}$. $50 \mathrm{ng} / \mu \mathrm{L}$ in $5 \mathrm{mM}$ Tris, $\mathrm{pH}$ 8.5. A final, more accurate, fluorometric-based assessment of DNA concentration was then performed on all samples using the Qubit ${ }^{\oplus}$ dsDNA HS Assay Kit (Invitrogen, UK). Fluorescence measurements (20 $\mu \mathrm{L}$ volumes) were performed on a 96 well qPCR thermal cycler (Quantica, Techne, UK), with seabass DNA concentrations being derived from a calibration curve generated from a set of standard dsDNAs. Based on these readings the seabass samples were diluted to $\mathrm{c}$. $10 \mathrm{ng} / \mu \mathrm{L}$ in $5 \mathrm{mM}$ Tris, $\mathrm{pH} 8.5$ for use in ddRAD library construction protocol.

\section{ddRAD library preparation and sequencing}

The ddRAD library preparation protocol used here is described in detail elsewhere [32, 33]. In silico estimation from the seabass genome predicted 52,230 ddRAD fragments with paired SbfI-SphI restriction site overhangs, while after the size selection applied in the present study (c. 320 bp -590 bp excluding adaptors) only 3603 fragments were predicted to be available.

Briefly, a single restriction enzyme digestion/adapter ligation reaction was performed for each progeny sample, while triplicate reactions were made for both dam and sire DNA samples. The latter ensured higher coverage of parental samples, which allowed more robust assignment of true SNPs in the pedigree. Each sample (40 ng DNA) was digested at $37^{\circ} \mathrm{C}$ for $30 \mathrm{~min}$ with $0.8 \mathrm{U}$ Sbfl ('rare' cutter, CCTGCA|GG motif) and $0.8 \mathrm{U}$ SphI ('common' cutter, GCATG|C motif) high fidelity restriction enzymes (New England Biolabs; NEB) in a $6 \mu \mathrm{L}$ reaction volume that included $1 \times \mathrm{CutSmart}^{\mathrm{Tm}}$ buffer (NEB). After cooling the reactions to room temperature, $3 \mu \mathrm{L}$ of a premade barcode-adapter mix was added to the digested DNA, and incubated at room temperature for $10 \mathrm{~min}$. This adapter mix comprised individual-specific barcoded combinations of P1 (Sbfl-compatible) and P2 (SphIcompatible) adapters at $6 \mathrm{nM}$ and $72 \mathrm{nM}$ concentrations respectively, in $1 \times$ reaction buffer 2 (NEB). Adapters were compatible with Illumina sequencing chemistry (see [31] for details). The barcoded adapters were designed such that adapter-genomic DNA ligations did not reconstitute RE sites, while residual RE activity limited concatemerization of genomic fragments during ligation. The adapters included an inline five- or seven-base barcode for sample identification (Additional file 1: Table S1). Ligation was performed over $40 \mathrm{~min}$ at $22{ }^{\circ} \mathrm{C}$ by addition of a further $3 \mu \mathrm{L}$ of a ligation mix comprising $4 \mathrm{mM}$ rATP (Promega, UK), and 2000 cohesive-end units of T4 ligase (NEB) in $1 \times$ CutSmart buffer.

The ligated samples were then heat denatured at $65{ }^{\circ} \mathrm{C}$ for $20 \mathrm{~min}$, cooled, and combined into a single pool. The pooled sample was column-purified (MinElute PCR Purification Kit, Qiagen, UK) and size selection of fragments, c. 320 bp to $590 \mathrm{bp}$, was performed by agarose gel electrophoresis. Following gel purification (MinElute Gel Extraction Kit, Qiagen, UK) the eluted size-selected template DNA (60 $\mu \mathrm{L}$ in EB buffer) was PCR amplified (11 cycles PCR; 28 separate $12.5 \mu \mathrm{L}$ reactions, each with $1 \mu \mathrm{L}$ template DNA) using a high fidelity Taq polymerase (Q5 Hot Start High-Fidelity DNA Polymerase, NEB). The PCR reactions were combined $(350 \mu \mathrm{L}$ total), and column-purified (MinElute PCR Purification Kit). The $55 \mu \mathrm{L}$ elute, in EB buffer, was then subjected to a further size-selection clean up using an equal volume of AMPure magnetic beads (Perkin-Elmer, UK), to maximize removal of small fragments (less than ca. $200 \mathrm{bp}$ ).

The final library was eluted in $20 \mu \mathrm{L}$ EB buffer and sequenced over two full Illumina MiSeq runs (v2 chemistry, 300 cycle kit, 162 bp paired end reads; Illumina, Cambridge, UK; 10.5 pM library applied and both runs spiked with $3 \%$ Illumina phiX control DNA). The raw sequence data from this study were deposited at the EBI Sequence Read Archive (SRA) with the accession number ERP006697.

\section{Genotyping ddRAD alleles}

Following initial analysis (FastQC: [34]) to confirm that high-quality sequence data had been generated, the MiSeq reads were processed using Stacks (v.1. 17; [35]), a package designed specifically to identify and score SNPs from restriction-enzyme based sequence data. First, the 'process_radtags' function was used to demultiplex the individual samples. During this process sequence reads with quality scores below 20 (-s set to 20), missing either restriction site or with ambiguous barcodes were discarded. Barcodes were removed and all sequences were 3' end trimmed to be 148 bases long. Then reference based Stacks analysis was performed, using 'ref_map.pl' perl script. Sequence alignment/map (SAM) files were created using Bowtie 2 aligner [36] and the seabass genome (dicLab v1; [16]). The main Stacks parameter values used in this analysis were $\mathrm{m}=10$ and $n=1$. In order to maximise the number of informative markers investigated 
while minimising missing or erroneous data, only polymorphic ddRAD-tags that containing 3 or less SNPs (maximum of 4 alleles) and which were detected in both parents and present in at least $75 \%$ of the offspring were scored.

\section{Genetic linkage map construction}

It was not feasible to construct a genetic linkage map de novo from the unordered meiotic gynogenetic family data. Both R/OneMap [37] and TMAP [38] were explored for genetic linkage map construction without success. The final map was constructed using R/OneMap after assigning markers to linkage groups based on the seabass genome assembly (see section $h$ below). Genotypes were imported in outcross format into R/OneMap in a modified way such that all genotypes shared the same segregation pattern ("ab x ab cross"). This package uses Hidden Markov Models (HMM) algorithms for outbred species while in parallel implements the methodology described in [39], for calculating the most probable linkage phase. Recombination fraction between all pairs of markers was calculated using rf.2pts function. These groups were ordered using the order.seq function in four available twopoint based algorithms including ser, $r c d, r e c$ and $\mu \mathrm{g}$ and the one which gave the smallest distance was selected for each LG. Following ordering, markers in the same LG were forced to the final map by using force function after inspection of safe order. The order of markers was also inspected visually using rf.graph.table which plots a heat map of LOD score and recombination frequency. Map distances were calculated in centiMorgans (cM) using the Kosambi mapping function. Genetic Mapper v0.5 [40] was used for the final visualisation of genetic linkage map of meiotic gynogenetic D.labrax.

\section{Visualising physical position of markers and microsatellites from previous studies}

Outputs of genome aligner (SAM files) were used for the positioning each ddRADseq locus and visualised using Genetic Mapper v0.5 [40]. Eleven microsatellite markers $[17,41]$ that have been used to differentiate between meiotic and mitotic gynogenetic sea bass [29] were also assigned to the physical map once the genomic positions were identified using Blastn $\left(10^{-20}\right.$ and lower).

\section{Marker-centromere mapping}

Centromeres are expected to be in regions with zero or low heterozygote frequency, with an increase in heterozygote frequency towards the telomeres. For each maternally informative ddRADseq locus, heterozygosity $(y)$ was computed across all progeny. Marker-centromere map distances (in $\mathrm{cM}$ ) were calculated using the formula $100 *(y / 2)$, under the assumption of complete interference, believed to be characteristic of fish species [4, 42, 43].

\section{Comparison of genomic assembly with linkage maps}

The genome assembly and the linkage map generated in the present study were compared to the recently published RAD-based high-density SNP-based genetic linkage map of Palaiokostas et al. [19], as an independent source for comparing marker order. Common polymorphic loci between the two linkage maps were identified by BLASTn. First, the loci beginning with the common enzyme recognition site motif ("TGCAGG"; Sbfl) from the present study (in total: 395 markers out of 764 female heterogametic assigned markers) were trimmed down to $95 \mathrm{bp}$, compatible with the RADseq P1 read length of [19]. Then a local nucleotide database was generated on Bioedit (version 7.2.5: [44]) from all assigned markers of [19] and all polymorphic markers of the present study were blasted against them. Stringent filtering options were applied to tabular output based on: i) e value $\leq 10^{-20}$, and ii) alignment length $\geq 90$ bases (i.e. at least $94.7 \%$ similarity).

\section{Estimation of recombination frequency per chromosome arm}

Scoring of homozygote/heterozygote distribution along the LGs of each individual progeny was used to estimate recombination, where a change from a region of homozygous markers (defined as at least two consecutive markers with the same status) to a region of heterozygous markers was taken as a crossover point. This analysis was carried out on 18 chromosomes where chromosome arm structure could be discerned (see Results).

\section{Results}

\section{ddRAD sequencing}

A total of $27,071,716$ paired-end raw reads were produced from the combined two sequencing runs for the meiotic gynogenetic $D$. labrax family with 79 progeny (Additional file 1: Table S1). Following demultiplexing using process_radtags, $77.1 \%$ of the raw paired-end reads were retained $(20,880,420)$. Only one sample offspring (MO241) failed to produce sufficient reads (c.1542 reads $<150 \mathrm{~K})$ and was dropped from subsequent STACKS analyses. As planned, the read numbers for both parents (785 K, sire \& $1127 \mathrm{~K}$, dam) exceeded those of offspring by a factor of c. 2 (average no. per reads per offspring, $504 \mathrm{~K})$. Read numbers for each sample are detailed in Additional file 2: Table S2. The reference-based Stacks analysis identified 6886 unique ddRAD loci and 1551 potential SNP loci (Fig. 1).

\section{Investigation of potential sire contribution}

Within the polymorphic marker dataset, 340 SNPs were identified with male-informative alleles, i.e. one (214 loci) or both (126 loci) alleles at a locus detected in the male parent alone. No male-specific alleles were detected in any of the offspring. Later mapping of these loci to the 


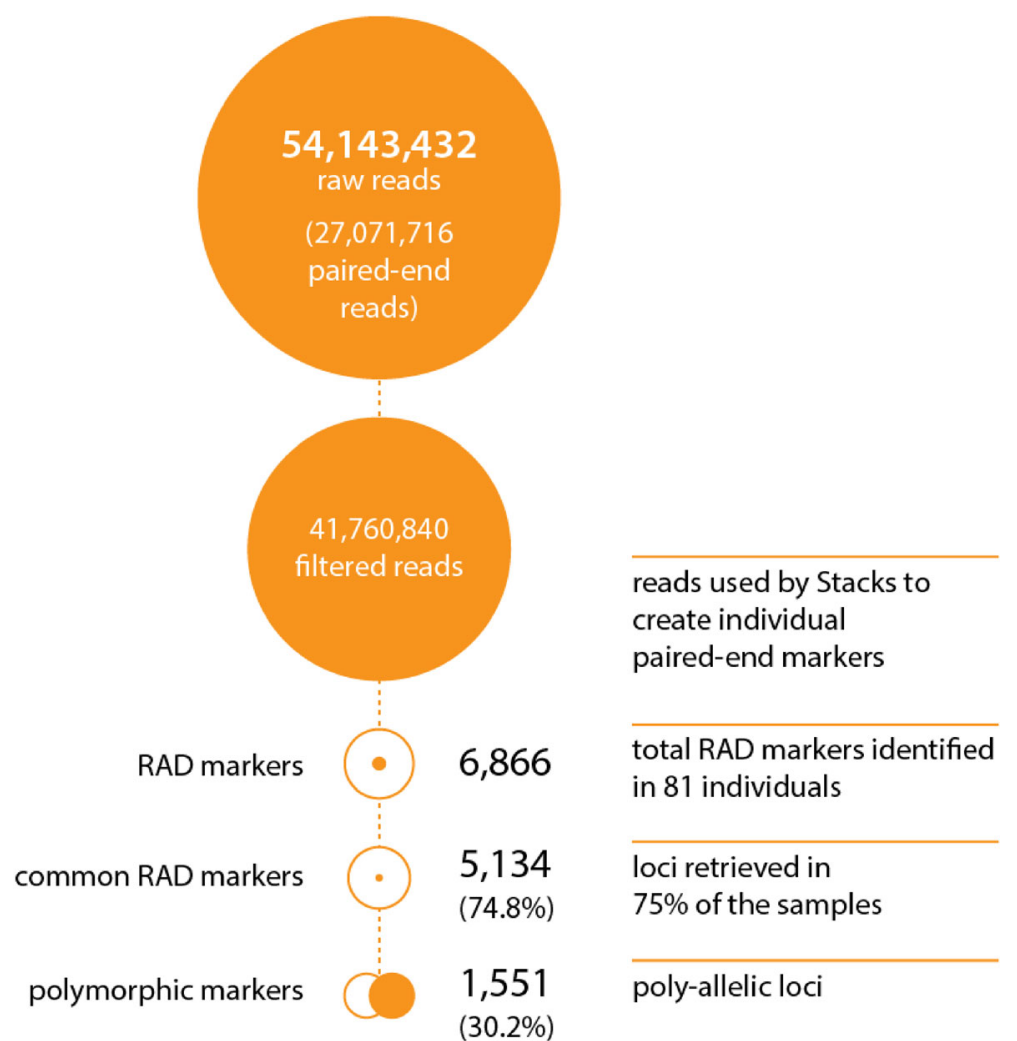

Fig. 1 Sequencing and ddRAD-tag summary. Detailed number of reads before and the after filters (orange disk) followed by the reconstructed numbers of ddRAD markers and polymorphic ddRAD markers (orange circles)

seabass reference genome confirmed that these markers were located across all seabass chromosomes. Thus no sire contribution was detected within the ddRAD dataset for this gynogenetic family.

\section{Construction of female genetic linkage map}

With the absence of paternal alleles confirmed, the marker dataset was refined to produce a robust set of informative SNPs for female map construction. Dam homozygous markers were removed (non-informative: 687 loci) as were loci where the minor allele frequency was $<0.4$ among the progeny samples (8 loci). Additionally 52 loci were removed since both parental genotypes were missing. This left data from 804 female-informative SNPs to be used in linkage map construction. The position of each SNP marker in the genome assembly is shown in Additional file 3: Table S3. The genomic position and informativeness of microsatellites used by [29] are shown in Additional file 4: Table S4, and both sets of markers are integrated into a physical map in Additional file 5: Fig. S1.

The linkage map (constructed using a LOD score of 4-5) comprised 764 SNPs and was $1251 \mathrm{cM}$ in length (Fig. 2; Table 1; Additional file 3: Table S3; Additional file 6: Dataset S1). Average marker distance was $1.63 \mathrm{cM}$ with 448 markers possessing unique positions. Linkage groups were between $23 \mathrm{cM}$ (LG 3) and $78 \mathrm{cM}$ (LG 1A) in length (mean $52 \mathrm{cM}$ ) and comprised between 15 (LG 18-21) and 46 markers (LG20; mean 32). As the initial grouping of SNPs within the linkage map was based on the genome assembly, the distribution of markers was in accordance with 24 chromosome pairs in D. labrax (originally identified from karyotype analyses; [45]).

\section{Marker-centromere mapping}

Heterozygote frequencies for 804 female informative markers in the meiotic gynogenetic family ranged between zero and one (i.e. 0 to $50 \mathrm{cM}$ map distances under the assumption of complete interference). Figure 3 shows a histogram of recombination frequencies and Additional file 7: Table S5 shows marker-centromere map distances. Seven loci $(0.87 \%$ of total loci) showed $100 \%$ recombination (i.e. telomeric), while 16 loci $(1.99 \%)$ showed zero recombination (i.e. centromeric). Almost half of the markers had heterozygote frequencies above 0.667 (49.12\%), the expected maximum theoretical value for independent segregation between a marker and the centromere when multiple crossovers occur, indicating high interference.

Eleven chromosomes (LG 1B, 2, 3, 6, 10, 11, 12, 15, $16,18-21$ and 20 ) showed single armed (mono-armed) behaviour, with heterozygosity rising from one end of 

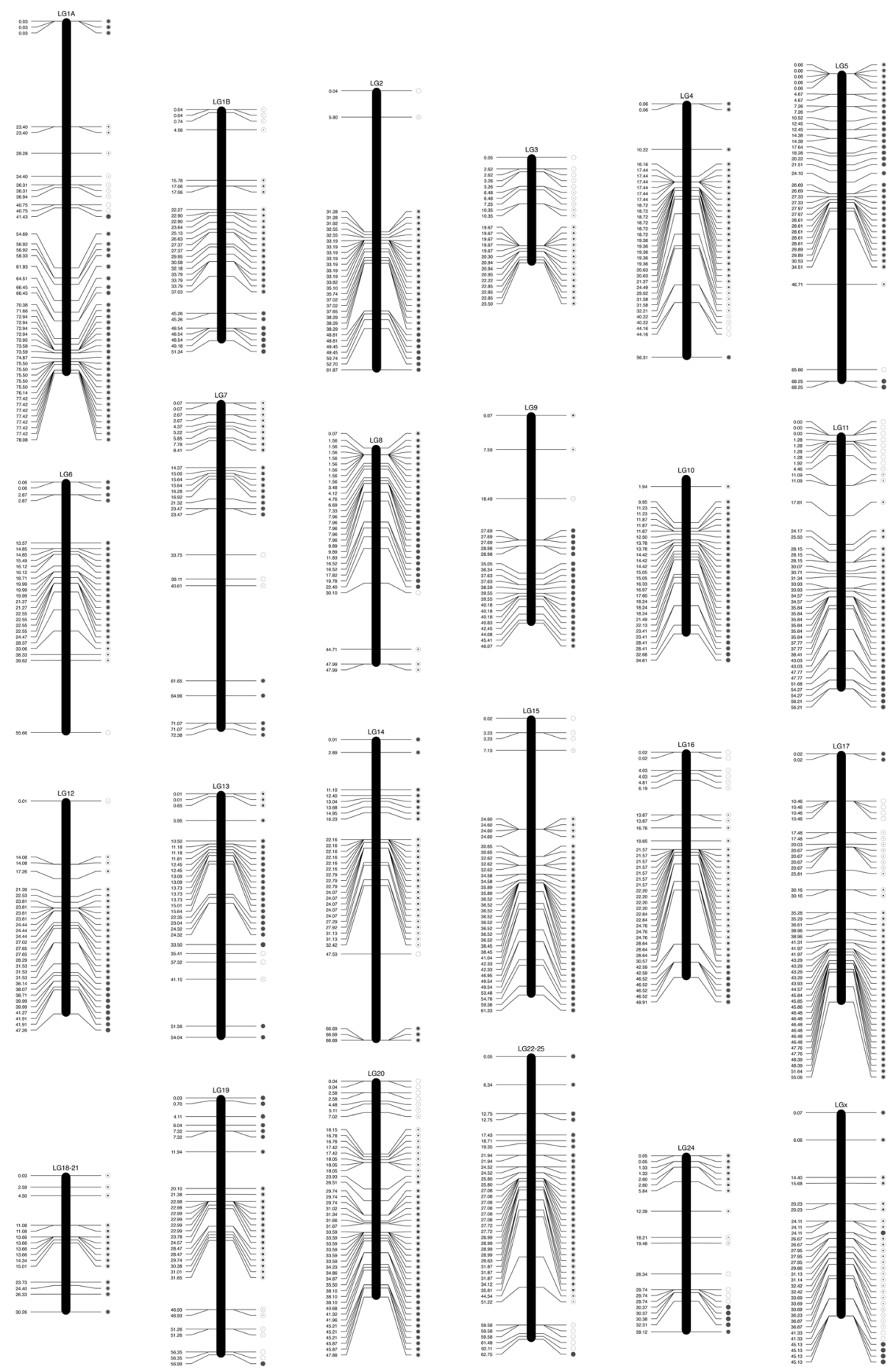

Fig. 2 Genetic linkage map of meiotic gynogenetic D. labrax. The positions on the left side of chromosomes are the distance in centiMorgans $(\mathrm{cM})$, the circles on the right hand side represent observed heterozygosity levels at each map position (empty circles represent homozygotes whereas increasingly filled black dots represents the higher levels of heterozygosity). Detailed data are provided in Additional file 3: Table S3

the chromosome to the other reaching up to almost $100 \%$. Figure 4 shows an example of crossover points in a mono-arm chromosome (LG11) in individual progeny, with the overall pattern for LG11. Three chromosomes (LG 4, 19 and 22-25) fitted the mono-armed pattern with the exception of a single outlying marker (i.e. the heterozygosity value for one marker did not fit the overall pattern). Three chromosomes (LG 14, 17 and 24) represented a clear bi-armed pattern (intermediate region with very low heterozygote frequency, rising towards a high 
Table 1 Summary of D. labrax genetic linkage map from a meiotic gynogenetic family, and assessment of chromosome structure

\begin{tabular}{|c|c|c|c|}
\hline LGs & No. of markers & Size $(c M)$ & Chromosome structure \\
\hline $\mathrm{LG} 1 \mathrm{~A}$ & 45 & 78.04 & ambiguous \\
\hline LG 1B & 29 & 51.30 & mono-arm \\
\hline LG 2 & 30 & 61.83 & mono-arm \\
\hline LG 3 & 24 & 22.79 & mono-arm \\
\hline LG 4 & 34 & 44.10 & mono-arm \\
\hline LG 5 & 38 & 68.19 & ambiguous \\
\hline LG 6 & 26 & 55.59 & mono-arm \\
\hline LG 7 & 26 & 72.31 & ambiguous \\
\hline LG 8 & 31 & 47.92 & ambiguous \\
\hline LG 9 & 23 & 46.00 & ambiguous \\
\hline LG 10 & 30 & 34.68 & mono-arm \\
\hline LG 11 & 42 & 54.26 & mono-arm \\
\hline LG 12 & 29 & 47.25 & mono-arm \\
\hline LG 13 & 27 & 54.03 & ambiguous \\
\hline LG 14 & 31 & 66.67 & bi-arm \\
\hline LG 15 & 37 & 61.31 & mono-arm \\
\hline LG 16 & 37 & 49.89 & mono-arm \\
\hline LG 17 & 45 & 55.03 & bi-arm \\
\hline LG 18-21 & 15 & 30.23 & mono-arm \\
\hline LG 19 & 30 & 56.96 & mono-arm \\
\hline LG 20 & 46 & 45.82 & mono-arm \\
\hline LG 22-25 & 39 & 62.70 & mono-arm \\
\hline LG 24 & 19 & 39.07 & bi-arm \\
\hline$(\mathrm{LGX})$ & 31 & 45.05 & assembly artefact \\
\hline Total & 764 & 1251.02 & \\
\hline
\end{tabular}

frequency at either end). One chromosome (LGX) fitted the bi-armed pattern with the exception of a single outlying marker (i.e. the heterozygosity value for one marker did not fit the overall pattern). Six chromosomes (LG 1A, $5,7,8,9$ and 13) did not show a clear pattern of heterozygosity along the chromosome that could enable us to assign an arm structure (mono-armed or bi-armed). This is summarised in Table 1.

To explore this further, we compared the RAD locus positions from the dense linkage map of Palaiokostas et al. [19] with those in the genome assembly. All of the linkage groups of the [19] map contained markers from the corresponding chromosome in the genome assembly, plus additional markers from unassigned (UNK) genome scaffolds. There were no cases where markers were assigned to different chromosomes in the assembly. The correlations for each linkage group are shown in Additional file 8: Table S6. The six LGs which did not show a clear pattern of heterozygosity in the current study were all among the 10 LGs showing the lowest correlation in marker order between the dense linkage map and the physical assembly, suggesting an association between the accuracy of the genome assembly and the clarity of arm structure derived from the present data. Of the 764 ddRADseq markers in the linkage map based on the meiotic gynogenetic family, 63 (8.2\%) were also found in the denser RADseq linkage map of [19]. All of these were found in the same linkage groups in both maps, and in the LGs with more than one such marker, the marker order in the present map corresponded to that of the denser map [19].

After removing the six chromosomes that did not show clear heterozygosity patterns (LG 1A, 5, 7, 8, 9 and 13) and the single anomalous markers in three chromosomes (LG 4, 19 and 22-25), the mean recombination frequency per chromosome arm was 0.989 (S.E. 0.123). However, there were instances of multiple crossovers (an

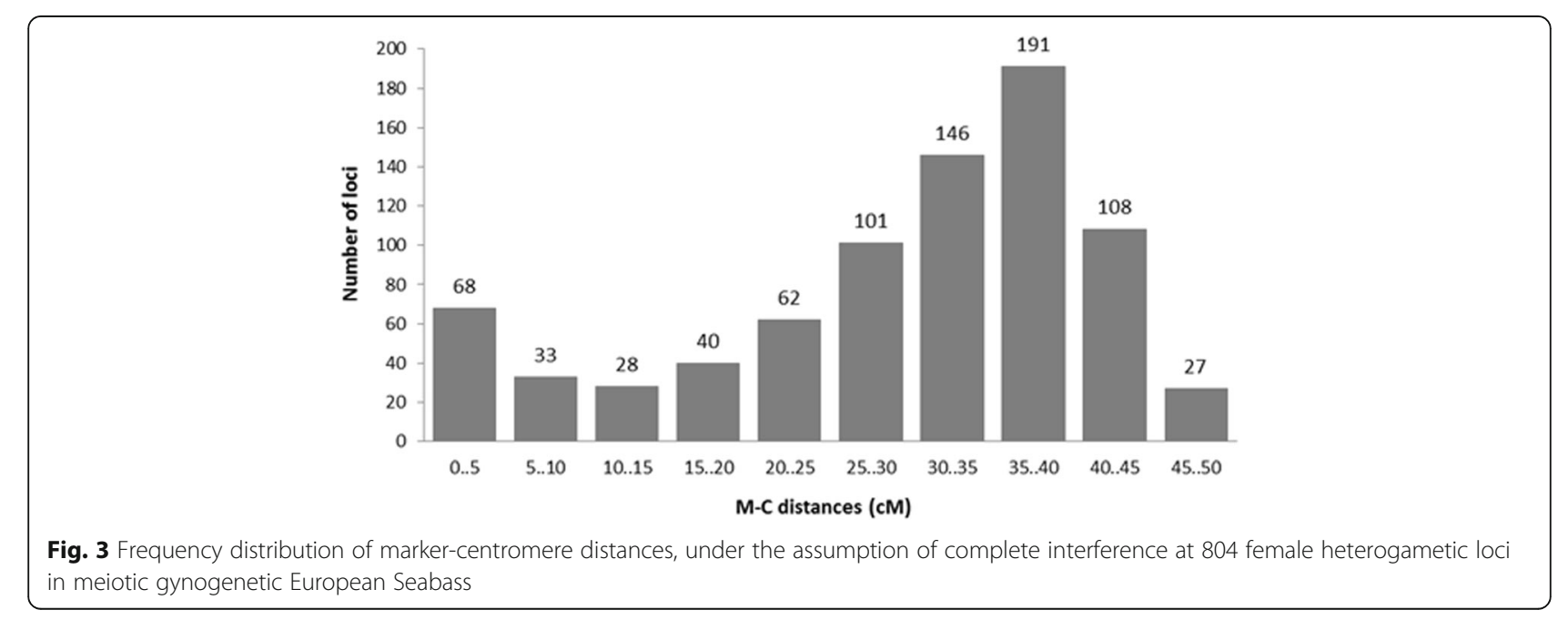




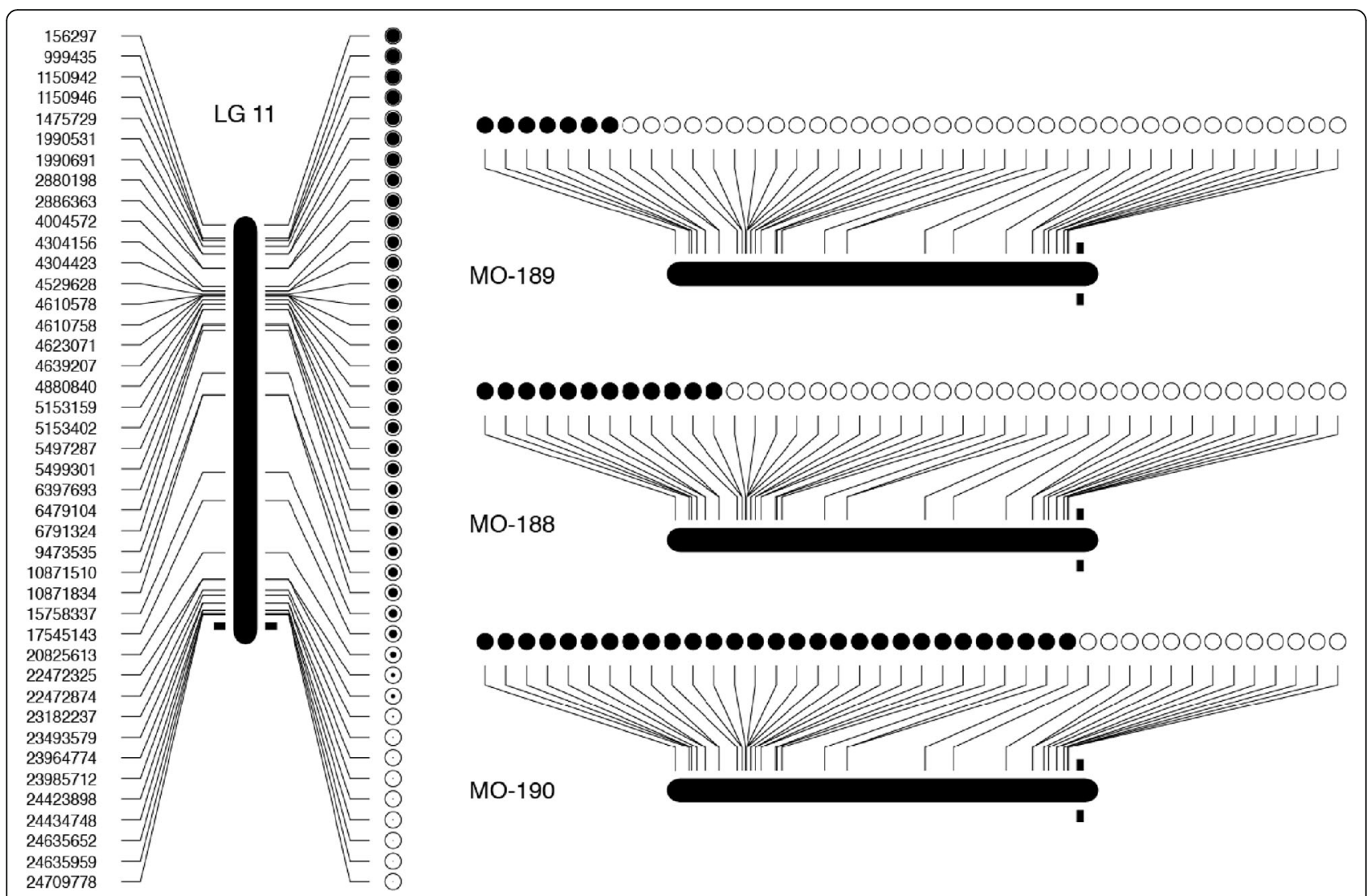

Fig. 4 Detailed example of recombination mapping in a single sea bass linkage group (LG 11), illustrating on the left side the computed recombination fraction for 79 progeny. Empty circles represent homozygotes close to the centromere (represented by black boxes either side of the linkage group), and increasingly filled black dots represent higher frequencies of heterozygotes towards the telomeric region. The panel to the right represents randomly chosen individuals from the meiotic gynogenetic family, showing the recombination points in LG 11

average of $11.36 \%$ overall) in some chromosome arms (see Additional file 9: Table S7).

\section{Discussion}

The present study constructed the first gene-centromere linkage map (of moderate marker density) for the European seabass, in order to identify markers at the distal end of the chromosomes. Such markers are more informative in discriminating between mitotic and meiotic gynogenetics, due to their higher recombination frequencies. Given the large number of markers showing high frequencies of heterozygotes in the meiotic gynogenetic family (almost half with $>67 \%$ heterozygotes, including seven with $100 \%$ heterozygotes), there would be a vanishingly small probability of mistaking a meiotic gynogenetic for a mitotic gynogenetic using such a marker set. This study also explored a second technical issue in the production of gynogenetic fish, that of potential paternal contribution following UV irradiation of sperm, by analysing large numbers of informative SNP markers (compared to smaller numbers of markers in previous studies on fish species).
The genotyping-by-sequencing approach used in this study (ddRADseq) proved to be very successful for both objectives, and also to be cost-effective for this purpose, generating 804 informative markers for the genecentromere map and 340 informative markers for assessing potential paternal contribution, from the analysis of a single ddRADseq library (in two sequencing runs). It is feasible to prepare and sequence such a library in one to two weeks for relatively modest cost, and this technique could thus be used routinely in verifying the development of isogenic lines in this and other fish species. RADseq [46] and its derivative ddRADseq [31] have already been used for genetic linkage mapping in model and non-model organisms [47-51], studies on sex determination systems $[52,53]$ and QTL analysis [54].

A requisite for successful production of uniparental fish is the ability to completely inactivate the genetic material in the irradiated gametes. In this study, 340 male informative SNP markers were identified, none of which were detected in any of the 79 progeny. These markers were located across all 24 linkage groups, confirming a lack of paternal contribution at this level of resolution. It 
is clear that using this protocol [23] we were able to produce a robust gynogenetic family, suitable for genecentromere mapping.

A genetic linkage map, comprising 764 SNPs spanning $1251.02 \mathrm{cM}$ with an average marker distance of $1.63 \mathrm{cM}$, was constructed. Approximately $95 \%$ of the femaleinformative SNPs (764 out of 804) were successfully placed on the linkage map. The genetic linkage map constructed in the present study was shorter than the denser map produced by [19], which had a total length of $4816 \mathrm{cM}$. The length of D. labrax linkage groups in the present study varied from $22.79 \mathrm{cM}$ to $78.04 \mathrm{cM}$ and exhibited a positive correlation, in most cases, with the number of markers mapped per linkage group. Markercentromere frequencies ranged between 0 and 1 ( 0 and $50 \mathrm{cM}$ ). These results clearly demonstrated that SNP loci produced by ddRAD sequencing were widely distributed in the seabass chromosomes, covering the entire chromosomal regions from proximal (centromeric) to distal (telomeric) regions. Theoretically under the assumption of no interference (with multiple crossover events potentially taking place between non-sister chromatids), the maximum frequency of heterozygotes should be $67 \%$ at the telomeres. However out of 804 female heterogametic SNP loci, 395 loci (49.12\%) showed heterozygote frequencies above 0.67 , indicative of crossover interference in seabass chromosomes. This phenomenon is well documented in the literature for other fish species $[4,8,43,55]$. Similar proportion of markers (48.1\%) with heterozygosity exceeding 0.67 were observed in turbot (Scophthalmus maximus) [56]. Twenty-seven of the seabass SNPs showed over $90 \%$ heterozygotes in the meiotic gynogenetic family (of which seven showed 100\% heterozygotes), suggesting that these could be used in individual SNP assays as a smaller scale assay for discriminating between meiotic and mitotic gynogenetics. At the centromeres of the chromosomes, 68 loci showed less than 10\% heterozygotes (of which 16 showed no heterozygotes). We detected at least four chromosomes that appeared to be bi-armed, rather more than the 0-2 biarmed chromosomes detected by karyotypic analysis (reviewed by [45]). The resolution of very small short chromosome arms from basic karyotyping is fairly poor, and it seems likely that applying large numbers of markers in studies such as the present one will result in the detection of more bi-armed chromosomes.

High levels of interference were reported in rainbow trout (Oncorhynchus mykiss) [4]. Subsequent literature suggests that high crossover interference is a wide-spread phenomenon in fish and shellfish species $[4,55-58]$. The results from the present study in general support this, with an average recombination frequency of around one per chromosome arm $(0.98 \pm 0.12$ (SE), see Additional file 5: Fig. S1). However some multiple crossovers were observed (an average of $11.36 \%$ ), suggesting that interference is not complete. The high marker density in this study probably helped to detect these events.

It was not possible to construct a genetic linkage map directly from the meiotic gynogenetic genotypic data in this study. It was not entirely clear if this was due to the nature of the data or the fact that linkage mapping softwares were not developed for this type of family. However, after defining linkage groups from the distribution of the markers in the sea bass genome assembly, we were able to order markers within these linkage groups with subsequent analyses, suggesting that this was a successful approach. We suggest that in any future similar studies, it would be better to produce a diploid biparental family as well as a meiotic gynogenetic family from the same parents, then the recombination data could be overlaid onto the linkage map constructed from the biparental sibs, which should contain essentially the same set of markers. This approach was followed to some extent previously in a study on rainbow trout [59] $(n=60$ in meiotic gynogenetic family; $n=60+60$ in two F1 crosses between two isogenic lines), however the meiotic gynogenetic family was only used for finding intervals where centromeres were located in the duplicated genome of the rainbow trout from a limited number of loci (pers. comm., R.Guyomard). These authors did not describe any attempt to construct a linkage map from the meiotic gynogenetic data.

Isogenic lines are likely to be a valuable resource for research on genetic improvement of complex traits in aquaculture of European seabass, as has already been demonstrated in other fish species, principally the rainbow trout [2]. Androgenesis appears to be an attractive route towards developing isogenic lines, and should lack the complication of spontaneous meiotic gynogenetics. However, the major problems encountered in inducing androgenesis in sea bass using UV irradiation of eggs [21], and indeed the paucity of publications on successful induction of androgenesis in other marine teleosts $[2,60]$, suggest that mitotic gynogenesis is currently the more likely successful route towards isogenic line development in this species.

\section{Conclusions}

In an effort to define telomeric markers to aid in the reliable production of isogenic lines by differentiating between meiotic and mitotic gynogenesis, we constructed a genetic linkage map and a gene-centromere map from a meiotic gynogenetic family of European seabass. This is the first genetic linkage map based on a meiotic gynogenetic family, although it was not possible to construct this de novo, so the draft genome of the sea bass was used for initial definition of the linkage groups. While there was high congruence between the genetic map from this study and the higher density map of Palaiokostas et al. 
[19], six linkage groups showed a lack of clarity in arm structure and low correlation in marker order between the dense linkage map of [19] and the genome assembly. This may reflect issues in the accurate assembly of these chromosomes in this first draft sea bass genome (dicLab v1). The data from the two linkage maps could be used in improving the genome assembly and interpreting the genomic data.

In the mapping family analysed, no paternal contribution was detected, validating the protocol used for UV inactivation of parental genome. The large number of telomeric and subtelomeric markers (i.e. those with high percentages of heterozygosity) in the meiotic gynogenetic family suggest that this approach should easily distinguish between meiotic and mitotic gynogenetics thus advancing/supporting future chromosomal set manipulation procedures in this species.

\section{Additional files}

Additional file 1: Table S1. Detailed information for each sample used: Sample ID, origin, UV irradiation and shock parameters, sampling tissue, fertilisation, sampling date and barcodes used per sample. (CSV 19 kb)

Additional file 2: Table S2. Comparison of ddRADseq runs (1st, 2nd and combined 1st +2 2nd sequencing runs). (CSV $5 \mathrm{~kb}$ )

Additional file 3: Table S3. All SNP markers used: Marker ID, locations of markers on physical map, genetic map corresponding of LGs, distance (CM) and the percentage of heterozygosity ratio. (CSV $27 \mathrm{~kb}$ )

Additional file 4: Table S4. Position of microsatellites from previous studies and their informative level. (CSV $1 \mathrm{~kb}$ )

Additional file 5: Fig. S1. Physical map of SNP and microsatellite markers in the European seabass genome. (PNG $2440 \mathrm{~kb}$ )

Additional file 6: Dataset S1. Marker ID, physical map location, percentage recombination frequency and sequences (FASTA format). (FASTA $132 \mathrm{~kb}$ )

Additional file 7: Table S5. Marker-centromere recombination rate (y) and map distances of 804 female heterogametic loci examined in meiotic gynogenetic seabass family. (CSV $45 \mathrm{~kb}$ )

Additional file 8: Table S6. Correlation of marker order in genome assembly with that of genetic linkage map of Palaiokostas et al. [19]; $n$ refers to number of markers in common between genome assembly and map of [19]. *:LGs with "ambiguous" arm structure based on heterozygosity pattern in the present study (see main text). (CSV 484 bytes)

Additional file 9: Table S7. Crossover points per chromosome arm. (CSV $3 \mathrm{~kb}$ )

\section{Abbreviations}

cM: CentiMorgan; ddRADseq: Double-digest Restriction-site Associated DNA sequencing; LG: Linkage Group; LOD: Logarithm of the odds; SNP: Single Nucleotide Polymorphism

\section{Acknowledgments}

Stéphane Lallement is acknowledged for identification and management of appropriate broodstock for the production of the gynogenetic family.

\section{Funding}

We gratefully acknowledge support from the European Fund Aquaculture infrastructures for excellence in European fish research, AQUAEXCEL project (FP7-262336), and from the MASTS pooling initiative (The Marine Alliance for Science and Technology for Scotland), funded by the Scottish Funding Council (grant reference HR09011) and contributing institutions. MO gratefully acknowledges the financial support of the Turkish Government (law 1416, YLSY), Ministry of Education for her PhD scholarship.

\section{Availability of data and materials}

The datasets supporting the conclusions of this article are included within the article and its additional files. The raw sequence data from this study were deposited at the EBI Sequence Read Archive (SRA) with the accession number ERP006697 (https://www.ebi.ac.uk/ena/data/view/PRJEB7015).

\section{Authors' contributions}

$\mathrm{MO}$ carried out extraction of genomic DNA, ddRAD library construction (under guidance of JBT), generated the genetic linkage map and the gene-centromere map, identified crossover points per linkage group, produced correlation graphs and comparison of genetic linkage maps with seabass genome assembly as well as data analysis and general statistics regarding regression. JC, MV, BC and SP produced the study material (meiotic gynogenetic $D$. labrax family). MB performed bioinformatics analysis: aligning ddRAD tags into genotypes, SNPs calling, visualising genetic linkage map from the marker order data provided by $\mathrm{MO}$, as well as advising on interpretation of the data and on bioinformatics. CP advised on interpretation of the data for genetic linkage map construction in the specific cross. HK and RR provided earlier access to draft genome of European seabass and advised on interpretation of the data. DJP and BJM conceived and designed the study and advised on interpretation of the data throughout. All authors read, edited and approved the manuscript.

\section{Authors' information}

MO (orcid.org/0000-0001-7318-6641)

MB (orcid.org/0000-0002-1206-7654)

DJP (orcid.org/0000-0001-8608-6631)

CP (orcid.org/0000-0002-4480-4612)

SP (orcid.org/0000-0002-9043-7438)

MV (orcid.org/0000-0001-9929-4587)

JBT (orcid.org/0000-0002-3843-9663)

\section{Competing interests}

The authors declare that they have no competing interests.

\section{Consent for publication}

Not applicable.

\section{Ethics approval and consent to participate}

The production of the gynogenetic family was performed in accordance with the French legislation on animal experiments, under the official animal experimentation license of B. Chatain (C 34-41, Level 1) approved by the Ministry for Agriculture, Agroalimentation and Forestry and in a certified laboratory (C 34-192-6) approved by the same Ministry. The research was also approved by the local ethical review body at the University of Stirling.

\section{Publisher's Note}

Springer Nature remains neutral with regard to jurisdictional claims in published maps and institutional affiliations.

\section{Author details}

'Institute of Aquaculture, School of Natural Sciences, University of Stirling, FK9 4LA Stirling, Scotland, UK. ${ }^{2}$ Cirad, Persyst, UMR Intrepid, Campus International de Baillarguet, 34398 Montpellier, France. ${ }^{3}$ Ifremer, 34250 Palavas-Les-Flots, France. ${ }^{4}$ INRA, GABI, AgroParisTech, Université Paris-Saclay, 78350 Jouy-en-Josas, France. ${ }^{5}$ Leibniz-Institute of Freshwater Biology and Inland Fisheries, Müggelseedamm 310, 12587 Berlin, Germany. ${ }^{6}$ Max-Planck-Institute for Plant Breeding, Max-Planck Genome Centre Cologne, Carl-von-LinnÃ@-Weg 10, D-50829 Cologne, Germany. 'Department of Arctic and Marine Biology, Faculty of Biosciences, Fisheries and Economics, University of Tromsø, 9037, Breivika, Tromsø, Norway.

Received: 22 December 2016 Accepted: 28 May 2017

Published online: 07 June 2017

\section{References}

1. Piferrer F, Beaumont A, Falguière JC, Flajšhans M, Haffray P, Colombo L. Polyploid fish and shellfish: production, biology and applications to 
aquaculture for performance improvement and genetic containment. Aquaculture. 2009;293:125-6.

2. Komen J, Thorgaard GH. Androgenesis, gynogenesis and the production of clones in fishes: a review. Aquaculture. 2007;269:150-73.

3. Purdom CE. Genetic engineering by the manipulations of chromosomes. Aquaculture. 1983;33:287-300.

4. Thorgaard GH, Allendorf FW, Knudsen KL. Gene-Centromere mapping in rainbow trout: high interference over long map distances. Genetics. 1983;103:771-83

5. Ihssen PE, Mckay LR, McMillan I, Phillips RB. Ploidy manipulation and Gynogenesis in fishes: cytogenetic and fisheries applications. Trans Am Fish Soc. 1990;119:698-717.

6. Hulata G. Genetic manipulations in aquaculture: a review of stock improvement by classical and modern technologies. Genetica. 2001;111:155-73.

7. Gomelsky B. Chromosome set manipulation and sex control in common carp: a review. Aquat Living Resour. 2003;16:408-15.

8. Danzmann RG, Gharbi K. Gene mapping in fishes: a means to an end. Genetica. 2001;111:3-23.

9. Nichols KM, Young WP, Danzmann RG, Robison BD, Rexroad C, Noakes M, et al. A consolidated linkage map for rainbow trout (Oncorhynchus mykiss). Anim Genet. 2003;34:102-15.

10. Brawand D, Wagner CE, Li YI, Malinsky M, Keller I, Fan S, et al. The genomic substrate for adaptive radiation in African cichlid fish. Nature. 2014:513:375-81.

11. Lien S, Koop BF, Sandve SR, Miller JR, Kent MP, Nome T, et al. The Atlantic salmon genome provides insights into rediploidization. Nature. 2016;533:200-5.

12. Bongers ABJ, Sukkel M, Gort G, Komen J, Richter CJJ. Development and use of genetically uniform strains of common carp in experimental animal research. Lab Anim. 1998;32:349-63.

13. Muller-Belecke A, Horstgen-Schwark G. Performance testing of clonal Oreochromis niloticus lines. Aquaculture. 2000;184:67-76.

14. Chourrout D, Quillet E. Induced gynogenesis in the rainbow trout : sex and survival of progenies, production of all-triploid populations. Theor Appl Genet. 1982;63:201-5.

15. Preston AC, Taylor JF, Craig B, Bozzolla P, Penman DJ, Migaud H. Optimisation of triploidy induction in brown trout (Salmo trutta L.). Aquaculture. 2013:414-5.

16. Tine M, Kuhl H, Gagnaire PA, Louro B, Desmarais E, Martins RST, et al. European sea bass genome and its variation provide insights into adaptation to euryhalinity and speciation. Nat Commun. 2014;5:5770.

17. Chistiakov DA, Hellemans B, Haley CS, Law AS, Tsigenopoulos CS, Kotoulas $\mathrm{G}$, et al. A microsatellite linkage map of the European sea bass Dicentrarchus labrax L. Genetics. 2005;170:1821-6.

18. Chistiakov DA, Tsigenopoulos CS, Lagnel J, Guo YM, Hellemans B, Haley CS, et al. A combined AFLP and microsatellite linkage map and pilot comparative genomic analysis of European sea bass Dicentrarchus labrax L. Anim Genet. 2008;39:623-34.

19. Palaiokostas C, Bekaert M, Taggart JB, Gharbi K, McAndrew BJ, Chatain B, et al. A new SNP-based vision of the genetics of sex determination in European sea bass (Dicentrarchus labrax). Genet Sel Evol. 2015;47:68.

20. Guyon R, Senger F, Rakotomanga M, Sadequi N, Volckaert FAM, et al. A radiation hybrid map of the European sea bass (Dicentrarchus labrax) based on 1581 markers: Synteny analysis with model fish genomes. Genomics. 2010;96:228-38.

21. Colléter J, Penman DJ, Lallement S, Fauvel C, Hanebrekke T, Osvik RD, et al. Genetic inactivation of European sea bass (Dicentrarchus labrax L.) eggs using UV-irradiation: observations and perspectives. PLoS One. 2014;9:e109572.

22. Bertotto D, Cepollaro F, Libertini A, Barbaro A, Francescon A, Belvedere P, et al. Production of clonal founders in the European sea bass, Dicentrarchus labrax L., by mitotic gynogenesis. Aquaculture. 2005;246:115-24.

23. Peruzzi S, Chatain B. Pressure and cold shock induction of meiotic gynogenesis and triploidy in the European sea bass, Dicentrarchus labrax L.: relative efficiency of methods and parental variability. Aquaculture. 2000;189:23-37.

24. Francescon A, Barbaro A, Bertotto D, Libertini A, Cepollaro F, Richard J, et al. Assessment of homozygosity and fertility in meiotic gynogens of the European sea bass (Dicentrarchus labrax L.). Aquaculture. 2005;243:93-102.

25. Yamamoto E. Studies on sex-manipulation and production of cloned populations in hirame, Paralichthys olivaceus (Temminck et Schlegel). Aquaculture. 1999;173:235-46.

26. Kato K, Hayashi R, Yuasa D, Yamamoto S, Miyashita S, Murata O, et al. Production of cloned red sea bream, Pagrus major, by chromosome manipulation. Aquaculture. 2002;207:19-27.
27. Braasch I, Postlethwait JH. Polyploidy in fish and the Teleost genome duplication. In: Soltis PS, Soltis DE, editors. Polyploidy and genome evolution. Berlin, Heidelberg: Springer; 2012. p. 341-83.

28. Havelka M, Bytyutskyy $D$, Symonová R, Ráb P, Flajšhans $M$. The second highest chromosome count among vertebrates is observed in cultured sturgeon and is associated with genome plasticity. Genet Sel Evol. 2016;48:12.

29. Colléter J. Towards the development of clonal lines in the Europeans seabass (D. labrax L.): application of uniparental reproduction techniques with insights into seabass eggs. PhD thesis, 2015, University of Montpellier 2.

30. Davey JW, Hohenlohe PA, Etter PD, Boone JQ, Catchen JM, Blaxter ML. Genome-wide genetic marker discovery and genotyping using nextgeneration sequencing. Nat Rev Genet. 2011;12:499-510.

31. Peterson BK, Weber JN, Kay EH, Fisher HS, Hoekstra HE. Double digest RADseq: an inexpensive method for de novo SNP discovery and genotyping in model and non-model species. PLoS One. 2012;7:e37135.

32. Manousaki T, Tsakogiannis A, Taggart JB, Palaiokostas C, Tsaparis D, Lagnel J, et al. Exploring a Nonmodel Teleost genome through RAD sequencinglinkage mapping in common Pandora, Pagellus erythrinus and comparative genomic analysis. Genes, Genomes, Genetics. 2016;6:509-19.

33. Brown JK, Taggart JB, Bekaert M, Wehner S, Palaiokostas C, Setiawan AN, et al. Mapping the sex determination locus in the hāpuku (Polyprion oxygeneios) using ddRAD sequencing. BMC Genomics. 2016;17:448.

34. Andrews S. FastQC: a quality control tool for high throughput sequence data, 2010. Available at: http://www.bioinformatics.babraham.ac.uk/projects/ fastqc. Accessed 3 Feb 2017.

35. Catchen JM, Amores A, Hohenlohe P, Cresko W, Postlethwait JH. Stacks: building and genotyping loci de novo from short-read sequences. Genes, Genomes, Genetics. 2011;1:171-82.

36. Langmead B, Salzberg S. Fast gapped-read alignment with bowtie 2. Nat Methods. 2013;9:357-60.

37. Margarido GRA, Souza AP, Garcia AAF. OneMap: software for genetic mapping in outcrossing species. Hereditas. 2007;144:78-9.

38. Cartwright DA, Troggio M, Velasco R, Gutin A. Genetic mapping in the presence of genotyping errors. Genetics. 2007;176:2521-7.

39. Wu R, Xing Ma C, Wu SS, Zeng Z. Linkage mapping of sex-specific differences. Genet Res. 2002;79:85-96.

40. Bekaert M. Genetic-Mapper: vectorial genetic map drawer. 2015. Available at: https://github.com/pseudogene/genetic-mapper. Accessed 3 Feb 2017.

41. García De León FJ, Dallas JJ, Chatain B, Canonne M, Versini JJ, Bonhomme F. Development and use of microsatellite markers in sea bass, Dicentrarchus labrax (Linnaeus, 1758) (Perciformes: Serranidae). Mol Mar Biol Biotechnol. 1995:4:62-8.

42. Sakamoto T, Danzmann RG, Gharbi K, Howard P, Ozaki A, Khoo SK, et al. A microsatellite linkage map of rainbow trout (Oncorhynchus mykiss) characterized by large sex-specific differences in recombination rates. Genetics. 2000;155:1331-45.

43. Nomura $\mathrm{K}$, Morishima $\mathrm{K}$, Tanaka H, Unuma T. Microsatellite - centromere mapping in the Japanese eel (Anguilla japonica) by half-tetrad analysis using induced triploid families. Aquaculture. 2006;257:53-67.

44. Hall AT. BioEdit: a user-friendly biological sequence alignment editor and analysis program for windows 95/98/NT. Nucleic Acids Symp Ser. 1999;41:95-8.

45. Sola L, Bressanello S, Rossi AR, laselli V, Crosetti D, Cataudella S. A karyotype analysis of the genus Dicentrarchus by different staining techniques. J Fish Biol. 1993;43:329-37.

46. Baird N, Etter PD, Atwood TS, Currey MC, Shiver AL, Lewis ZA, et al. Rapid SNP discovery and genetic mapping using sequenced RAD markers. PLoS One. 2008;3:e3376

47. Anderson JL, Rodri AR, Braasch I, Amores A, Hohenlohe P, Batzel P, et al. Multiple sex-associated regions and a putative sex chromosome in zebrafish revealed by RAD mapping and population genomics. PLoS One. 2012;7:e40701.

48. Recknagel H, Elmer KR, Meyer A. A hybrid genetic linkage map of two ecologically and morphologically divergent Midas cichlid fishes (Amphilophus spp.) obtained by massively parallel DNA sequencing (ddRADSeq). Genes, Genomes, Genetics. 2013, 3:65-74.

49. Gonen S, Lowe NR, Cezard T, Gharbi K, Bishop SC, Houston RD. Linkage maps of the Atlantic salmon (Salmo salar) genome derived from RAD sequencing. BMC Genomics. 2014;15:166.

50. Kai W, Nomura K, Fujiwara A, Nakamura Y, Yasuike M, Ojima N, et al. A ddRAD-based genetic map and its integration with the genome assembly of Japanese eel (Anguilla japonica) provides insights into genome evolution after the teleost-specific genome duplication. BMC Genomics. 2014;15:233. 
51. Palaiokostas C, Bekaert M, Khan MGQ, Taggart JB, Gharbi K, McAndrew BJ, et al. A novel sex-determining QTL in Nile tilapia (Oreochromis niloticus). BMC Genomics. 2015;16:171.

52. Palaiokostas C, Bekaert M, Davie A, Cowan ME, Oral M, Taggart JB, et al. Mapping the sex determination locus in the Atlantic halibut (Hippoglossus hippoglossus) using RAD sequencing. BMC Genomics. 2013;14:566.

53. Palaiokostas C, Bekaert M, Khan MGQ, Taggart JB, Gharbi K, McAndrew, B J, Penman DJ. Mapping and validation of the major sex-determining region in Nile tilapia (Oreochromis niloticus L.) using RAD sequencing. PLoS One 2013;8:e68389.

54. Houston RD, Davey JW, Bishop SC, Lowe NR, Mota-Velasco JC, Hamilton A, et al. Characterisation of QTL-linked and genome-wide restriction siteassociated DNA (RAD) markers in farmed Atlantic salmon. BMC Genomics. 2012;13:244

55. Morishima K, Nakayama I, Arai K. Microsatellite-centromere mapping in the loach, Misgurnus anguillicaudatus. Genetica. 2001;111:56-9.

56. Martínez P, Hermida M, Pardo BG, Fernández C, Castro J, Cal RM, et al. Centromere-linkage in the turbot (Scophthalmus maximus) through halftetrad analysis in diploid meiogynogenetics. Aquaculture. 2008;280:81-8

57. Reid DP, Smith CA, Rommens M, Blanchard B, Martin-Robichaud D, Reith M. A genetic linkage map of Atlantic halibut (Hippoglossus hippoglossus L.). Genetics. 2007:177:1193-205.

58. Nie H, Li Q, Kong L. Centromere mapping in the Pacific abalone (Haliotis discus hannai) through half-tetrad analysis in gynogenetic diploid families Anim Genet. 2012;43:290-7.

59. Guyomard R, Mauger S, Tabet-Canale K, Martineau S, Genet C, Krieg F, et al. A type I and type II microsatellite linkage map of rainbow trout (Oncorhynchus mykiss) with presumptive coverage of all chromosome arms. BMC Genomics. 2006;7:302

60. Hou JL, Wang GX, Zhang XY, Sun ZH, Liu HJ, Wang YF. Cold-shock induced androgenesis without egg irradiation and subsequent production of doubled haploids and a clonal line in Japanese flounder, Paralichthys olivaceus. Aquaculture. 2016;363:642-6.

\section{Submit your next manuscript to BioMed Central and we will help you at every step:}

- We accept pre-submission inquiries

- Our selector tool helps you to find the most relevant journal

- We provide round the clock customer support

- Convenient online submission

- Thorough peer review

- Inclusion in PubMed and all major indexing services

- Maximum visibility for your research

Submit your manuscript at www.biomedcentral.com/submit

) Biomed Central 